\title{
Escaping inhibition
}

DOI:

10.1038/nrd2253
The success of the ABL-kinase inhibitor imatinib in the treatment of BCR-ABL-driven leukaemia raised hopes that drugs that target key kinases underlying other cancers, such as members of the human epidermal growth factor receptor (HER) family, might be similarly efficacious. However, several smallmolecule inhibitors of HER family kinases have shown limited efficacy in HER2-driven breast cancers, despite effective inhibition of kinase activity. Writing in Nature, Sergina and colleagues now provide an explanation for this phenomenon: failure to completely inhibit the kinase activity of HER2 allows oncogenic signalling through the kinase-inactive family member HER3 to continue.

Signalling in the HER family, which consists of epidermal growth

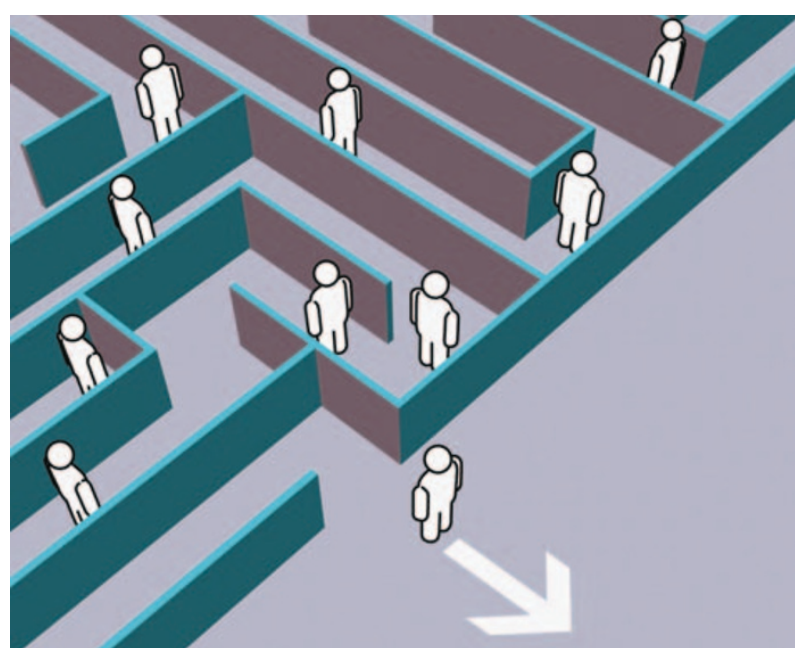

factor receptor (EGFR), HER2, HER3 and HER4, involves receptor dimerization and transphosphorylation, which leads to the activation of various pathways, including the potentially oncogenic phosphatidylinositol 3-kinase (PI3K)/Akt pathway. Given the abundance of preclinical and clinical data indicating the importance of EGFR- and HER2driven signalling in various cancers, drug discovery programmes have focused on developing tyrosine kinase inhibitors (TKIs) that target one or both of these kinases. However, although these agents are effective at inhibiting EGFR and HER2 phosphorylation in patients' tissues and tumours, Akt activity is not inhibited as might be anticipated in many patients, which could explain the limited clinical activity of the drugs.

To investigate this puzzle, Sergina et al. studied Akt inhibition by various HER TKIs in HER2-driven breast cancer cells in vitro and in tumour xenografts. Surprisingly, although Akt signalling was initially inhibited by the TKIs, this inhibition was not sustained. Assessment of signalling activity by HER family members revealed that although EGFR and HER2 phosphorylation and associated downstream pathways are durably inhibited by TKIs, dephosphorylation of HER3 is transient. This explains why Akt signalling is reactivated, because signalling in the $\mathrm{PI} 3 \mathrm{~K} /$ Akt pathway is driven primarily by transphosphorylation of HER3.
Further studies showed that inhibition of EGFR and/or HER2 by TKIs leads to compensatory changes in the HER3 phosphorylationdephosphorylation equilibrium, which seems to be driven by Akt-mediated negative-feedback signalling. The TKI-refractory HER3 phosphorylation is due to HER2 because it can be suppressed by anti-HER2 siRNA transfection. In addition, complete inactivation of HER2 with high doses of TKIs prevents Akt activity being restored by HER3 signalling.

So, it seems that in order to treat HER2-driven breast cancers more effectively, drugs that are able to fully inactivate HER2 kinases are needed to prevent oncogenic Akt signalling being reactivated by HER3. As the authors note, until compounds with the necessary potency and specificity can be found, combinations of agents designed to undermine the resiliency of HER-family signalling might represent the most promising therapeutic approach. Furthermore, whichever strategy is tested, HER3 transphosphorylation should be used as a biomarker to monitor treatment efficacy rather than inhibition of autophosphorylation, which is used at present.

Peter Kirkpatrick

ORIGINAL RESEARCH PAPER Sergina, N. V. et al. Escape from HER-family tyrosine kinase inhibitor therapy by the kinase-inactive HER3. Nature 7 Jan 2007 (doi: 10.1038/nature05474) 